\title{
Adult Intussusception: Three Cases Reports and Review of the Literature.
}

\author{
Amal Hajri, Karim Yaquine, Saad Rifki El Jai, Rachid Boufettal, Driss Errguibi, and Farid Chehab
}

\begin{abstract}
Adult intussusception occurs infrequently and differs from childhood intussusception in its presentation, etiology, and treatment. Diagnosis can be delayed because of its longstanding, intermittent, and non-specific symptoms and most cases are diagnosed at emergency laparotomy. Treatment entails simple bowel resection in most cases. Reduction of the intussusception before resection is controversial, but there is a shift against this, especially in colonic cases. This paper presents the diagnosis and management of three cases of adult intussusception, in our hospital.
\end{abstract}

Index Terms- adult; bowel obstruction; intussusception.

\section{INTRODUCTION}

Intestinal intussusception in adults is rare (about $0.003 \%$ $0.02 \%$ of all hospital admissions). It has traditionally been considered associated with an underlying cause in about $90 \%$ of cases [1]. Intussusception occurs when one portion of the gut becomes invaginated within an immediately adjacent segment, almost invariably, proximal into the distal [2]. The clinical presentation of intussusception in adults is variable in appearance. The signs and symptoms of the bowel obstruction predominate in $82 \%$ of cases. [3] The diagnosis in adults is usually made at laparotomy, as most patients present as an emergency with intestinal obstruction. In non-emergency patients the diagnosis can be challenging as symptoms include intermittent abdominal pain that often settles comparatively quickly. [4]. we report three cases of adult intussusception.

\section{Case report 1}

We report the case of a 43-year-old patient, chronic smoking, admitted to the emergency room for acute abdominal pain associated with rectrorrhagia of average abundance of acute installation evolving in a context of apyrexia and preservation of the general state. The clinical examination objectified a diffuse abdominal tenderness with digital rectal examination, palpation of the intestine by the pulp of the finger with the presence of red blood. The abdominal CT was in favor of a sigmoid-rectal invagination (Figure 1) without signs of detectable gravity.

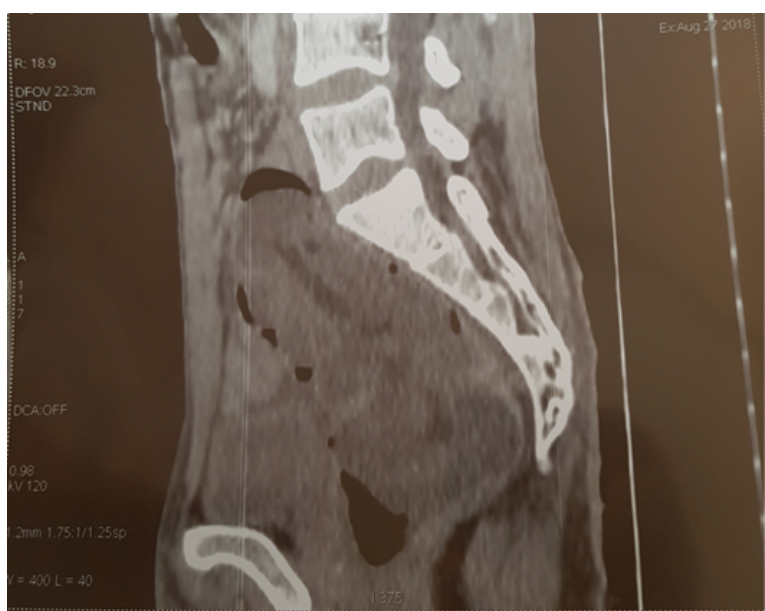

Fig. 1. Sagittal plain CT scan of the abdomen showing colorectal intussusception.

The intervention consisted of an ileo-hemi-colectomy with an ileo-colic end-lateral anastomosis. Surgical exploration objectified an ileo-colonic invagination involving 1 meter of hail as well as the right colon which are invaginated at the level of the left colon on an anomaly of rotation of the hail realizing the aspect of a complete common mesentery (Figure2). Given the extent of the invagination, we had proceeded to a disinvagination before the resection (Figure 3), having objectified a thickened cardboard aspect of the cecal bottom with a flow of lymphadenopathies along the ileo-coeco-colo-appendicular artery (Figure 4). The post-operative suites were simple. The anatomopathological examination of the operating room was in favor of ischemic and hemorrhagic colonic necrosis without signs of specificity or malignancy.

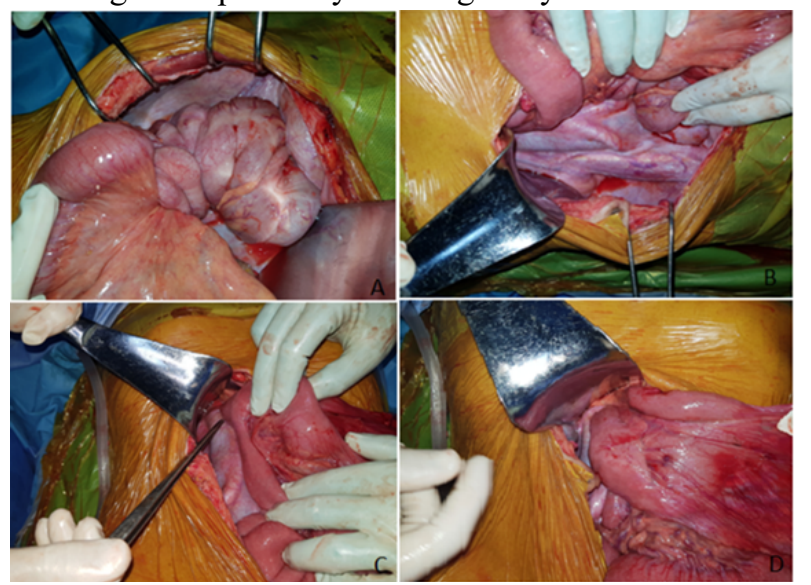

Fig. 2. Intraoperative image showing: A: The ileocolic intussusception; B; C; D: The anomaly of rotation of the hail realizing the aspect of a complete common mesentery. 


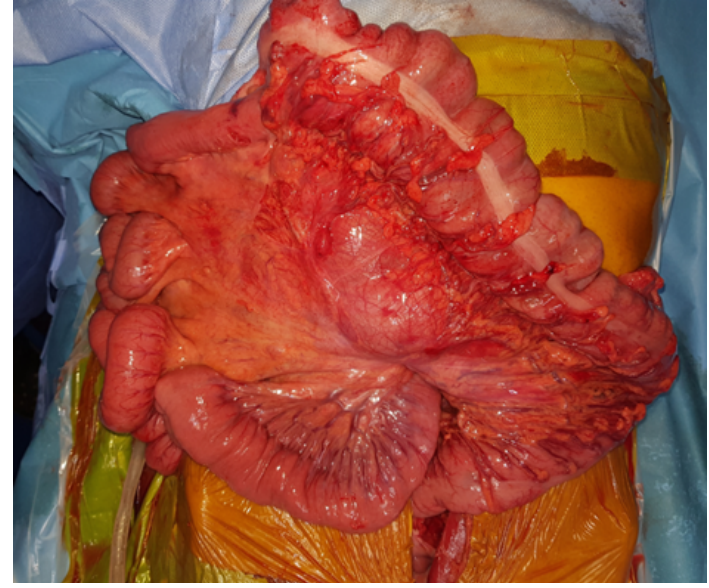

Fig. 3. Intussusception being reduced intra-operatively showing the anomaly of rotation of the hail realizing the aspect of a complete common mesentery.

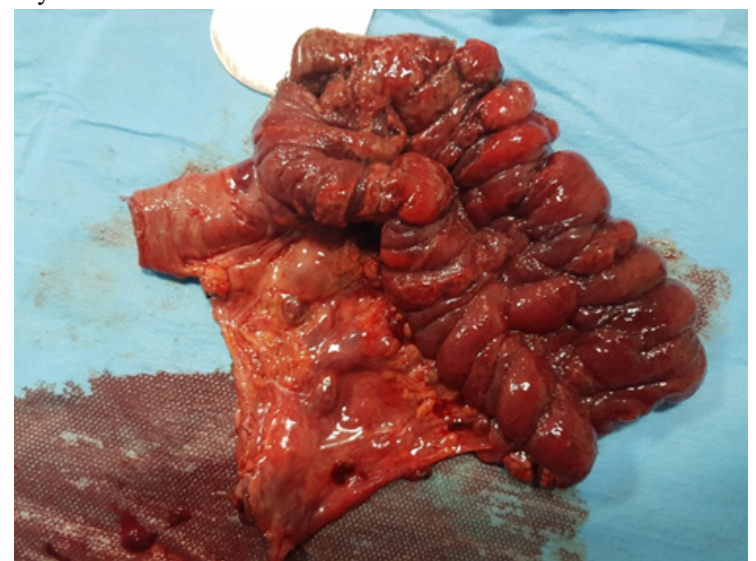

Fig. 4. Photograph showing when the intramuscular lumen was opened after right hemi colectomy.

\section{Case report 2}

Patient aged 68 years, cholecystectomized 2 years ago by laparoscopy, who presents 1 month before his admission of rectorrhagia of medium abundance, associated with chronic constipation. The clinical examination was unremarkable with a normal digital rectal exam. Colonoscopy had objectified an ulcero-budding process of the sigmoid at 28 $\mathrm{cm}$ from the anal margin extended over $10 \mathrm{~cm}$. with an anatomopathology a tubulo-villous adenoma mainly in dysplasia of low grade and focal in dysplasia of high grade. The abdominal CT was in favor of a parietal thickening of the sigmoid loop of tumor appearance associated with a locoregional lymphatic attenuation of the mesosigmoid and inferior mesenteric. diffuse parietal thickening of the inflammatory-looking rectum associated with densification of pelvic fat.

The intervention consisted of an anterior colorectal resection with an end-to-end colorectal anastomosis, a protective ileostomy with drainage of the Douglas CDS by Salem probe. Surgical exploration objectified colorectal invagination on the sigmoid tumor process without secondary localization. The post-operative suites were simple. The anatomopathological examination was in favor of a moderately differentiated and infiltrating adenocarcinoma classified pT2N0M0.

\section{Case report 3}

A 50-year-old patient with no particular pathological history, who presented to the emergency department for pain in the left iliac fossa, fever and rectorrhagia of low abundance. The abdominal examination had objectified a pain of the left iliac fossa, and with the rectal examination, palpation of the pudding of invagination

The abdominopelvic CT had objectified a recto-sigmoid invagination with sigmoid glides in intra rectal in intraluminal accompanied by the mesenteric fat extended on $15 \mathrm{~cm}$ with thickened rectal wall, hypodense edema, associated with parietal pneumatosis witnessing of a digestive suffering. An irregular digestive thickening measuring approximately $36 \mathrm{~mm}$ is individualized within it (Figure 5 and 6). The surgical indication was asked but the patient refused to be operated.

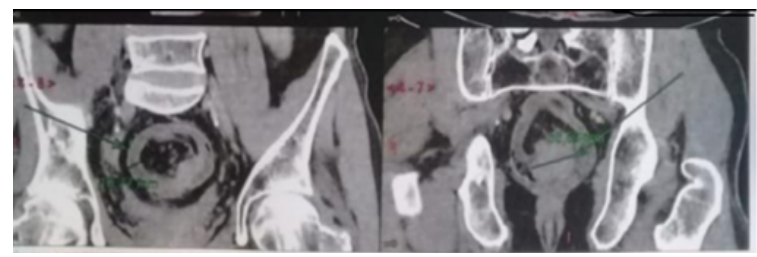

Fig. 5. Coronal reconstruction demonstrating the colorectal intussusception, with an irregular digestive thickening is individualized within it.

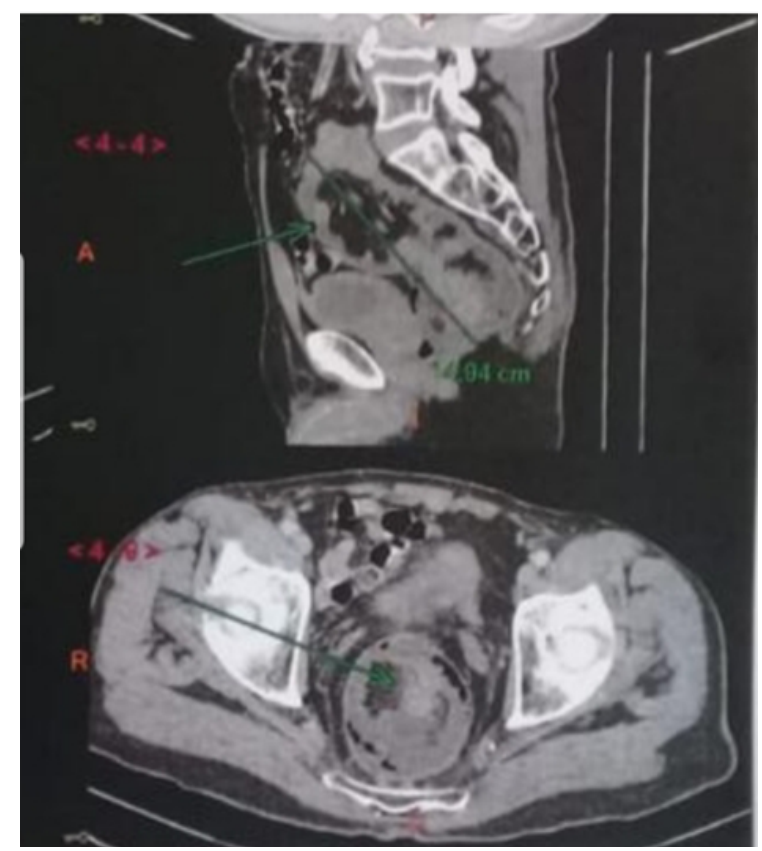

Fig. 6. Scan images of the abdomen demonstrating the colocolic intussusception. A) Sagittal and B) plain axial CT.

\section{DISCUSSION}

Intussusception is the invagination of a bowel loop (intussusceptum) with its mesenteric fold into the lumen of a contiguous portion of bowel (intussuscipiens) as a result of peristalsis. It is generally believed that masses in the bowel or lumen act as an irritant and provoke abnormal peristaltic movement, which may lead to the telescoping of one bowel segment over the adjacent segment [5]. Adult intussusception is a rare entity encountered by surgeons. It differs from intussusception in children in various aspects, regarding etiology, clinical presentation, diagnostic approach, and management. Intussusception has been classified into 4 categories according to the site of origin, such as enteric, ileocolic, ileocecal, and colocolic [6]. The presenting symptoms in adult patients with intussusceptions 
are non-specific and often long standing. Most series report pain as the commonest symptom, being present in $71 \%$ to $90 \%$ of patients, with vomiting and bleeding from the rectum as the next most common symptoms [4]. In our study, all the patients present bleeding.

However, while until the last few years, intussusception was diagnosed mainly in the operating room, the growing application of $\mathrm{CT}$ for abdominal imaging, in many clinical situations, has led to increased detection of transient intussusceptions without an underlying disease $[1,7,8,9]$. In our study, the abdominopelvic CT had objectified the intussusception in two cases. In the third case the diagnostic had established at per operative.

Almost $90 \%$ of adult cases are due to an underlying pathological lead point including malignant or benign neoplasms, intestinal polyps, Meckel's Diverticulum, postoperative adhesions, inflammatory bowel disease, motility disorders and iatrogenic causes such as gastric tubes $[10,11,12]$. The remaining $10 \%$ cases are idiopathic. The majority of the adult colo-colonic intussusceptions caused by a pathological lead point are due to primary carcinomas $65-70 \%[12,13]$. In our study two cases are caused by colonic mass, in another case the intussusceptions are caused by an anomaly of rotation of the hail realizing the aspect of a complete common mesentery.

Adult intussusception warrants laparotomy rather than attempts at hydrostatic reduction in view of the high incidence of underlying abnormality. Controversy remains as to whether reduction of the intussuscepting lesion should be attempted at operation. Early reports advocated reducing the intussusception before resection. The perceived disadvantage of this is that malignant cells may be disseminated during the process despite no clear evidence on this issue. On the other hand, the advantages of reducing the intussusception especially when the small bowel is affected are that it may be possible to preserve considerable lengths of bowel and thereby prevent development of short bowel syndrome [4].

Begos et al [4, 14] suggest resection without attempting reduction when the bowel is inflamed, ischemic, or friable and in obvious colo-colic intussusception (given the high likelihood of malignancy). In all other cases reduction should always be attempted initially. However, Azar et al [4] suggested that surgical resection without reduction is the preferred treatment in adults, as almost $50 \%$ of both colonic and enteric intussusceptions are associated with malignancy. Simple reduction is however acceptable in post-traumatic and idiopathic intussusceptions where no pathological cause is usually present in the bowel $[4,15]$.

\section{CONCLUSION}

Intussusception is relatively rare in the adult population and this, along with the vague clinical features, makes diagnosis difficult. The CT scan has a major role in the diagnosis. Surgical treatment with resection is the optimal treatment option. However, if not managed in a timely fashion, intussusception may lead to significant morbidity which includes complete bowel obstruction, ischemia, gangrene, perforation, peritonitis and lifethreatening sepsis.

\section{REFERENCES}

[1] Silvia Tresoldi, 1 Young H. Kim, 2 Michael A. Blake, 3 Mukesh G. Harisinghani, 3 Peter F. Hahn, 3 Stephen P. Baker, 4 Peter R. Mueller, 3 Krishna Kandarpa2. Adult intestinal intussusception: can abdominal MDCT distinguish an intussusception caused by a lead point? Abdom Imaging (2007) DOI: 10.1007/s00261-007-9328-8.

[2] Anupam Das, Lalmalsawma Ralte, A. S. Chawla, S. V. Arya, Anil Kumar, Ravi Saroha, and Dheer Singh Kalwaniya Colo colic Intussusception in an Older Child: A Rare Case Report and a Literature Review Hindawi Publishing Corporation Case Reports in Surgery Volume 2013, Article ID 106831, 3 pages http://dx.doi.org/10.1155/2013/106831.

[3] Ivan Žokalj1, Zvonimir Magaš2, Zlatko Pavčec1, Hussein Saghir1, Andrej Pal1, Zvonimir Kolarić3, Miljenko Marotti4. Adult obstructing ileocolic intussusception Radiol Oncol 2007; 41(3): 10712. Doi: 10.2478/v10019-007-0018-5

[4] S Yalamarthi, R C Smith. Adult intussusception: case reports and review of literature. Postgrad Med J 2005; 81:174-177 . Doi: 10.1136/pgmj.2004.022749

[5] Bipradas Roy, Ramanuj Mukherjee, Susil Kumar Paira, Sandip K Halder, Gautam Bhaumik R G Kar Medical College, Calcutta, Indian Cer San D . Adult colocolic intussusception due to pedunculated lipoma-uncommon disease, common presentation; case report. (J Surg Arts): 2012; 5(2): 42-44.

[6] Jamel Saad, MD, Asem Hassan, MD, Nadeem Zafar, FRCP. Adult intussusception an overlooked diagnosis in the emergency department Hussein A. Mostafa, MD, Saudi Med J 2014; Vol. 35 (3)

[7] Takeuchi K, Tsuzuki Y, Ando T, et al. The diagnosis and treatment of adult intussusception. J Clin Gastroenterol 36:18-21(2003)

[8] Sandrasegaran K, Kopecky KK, Rajesh A, Lappas J. Proximal small bowel intussusceptions in adults: CT appearance and clinical significance. J Abdom Imaging 29 :653-657(2004).

[9] Lvoff N, Breiman RS, Coakley FV, Lu Y, Warren RS.Distinguishing features of self-limiting adult small-bowel intussusception identified at CT. Radiology 227:68-72(2003).

[10] Rutherford CL., et al. Colo-colic intussusception secondary to lipomatous polyp in an adult. BMJ Case Report (2013).

[11] Wang Y., et al. Adult colo-colonic intussusception caused by congenital bands: A case report and literature review. International Journal of Surgery Case Reports 26 (2016): 88- 89.

[12] Indran B Indrakrishnan., et al. A Rare Cause of Adult Colo-Colonic Intussusception: Colonic Lipoma. Acta Scientific Gastrointestinal Disorders 2.4 (2019): 06-09.

[13] Mouaqit O., et al. Pedunculated lipoma causing colo-colonic intussusception: a rare case report. BMC Surgery 13 (2013): 51.

[14] Begos DG, Sandor A, Modlin IM. The diagnosis and management of adult intussusception. Am J Surg 1997; 73:88-94.

[15] Kitamura K, Kitagawa S, Mori M, et al. Endoscopic correction of intussusception and removal of a colonic lipoma. Gastrointest Endosc 1990; 36:509-11

\section{Amal Hajri}

Casablanca, Morocco 20400

Telephone: 00212613299259

e-mail: amalhajri.282@gmaill.com

Institution: Department of General Surgery III, Ibn Rochd University Hospital, Casablanca, Morocco.

\section{Karim Yaquine}

e-mail: yaqine.karim@gmail.com

Institution: Department of General Surgery III, Ibn Rochd University Hospital, Casablanca, Morocco.

\section{Saad Rifki EI Jai}

e-mail: Saadjai@yahoo.fr

Institution: Department of General Surgery III, Ibn Rochd University Hospital, Casablanca, Morocco.

\section{Rachid Boufettal}

e-mail: racbouf@hotmail.fr

Institution: Department of General Surgery III, Ibn Rochd University Hospital, Casablanca, Morocco.

\section{Driss Errguibi}

e-mail: erguibi_d@yahoo.fr

Institution: Department of General Surgery III, Ibn Rochd University Hospital, Casablanca, Morocco. 


\section{Farid Chehab}

Head of the digestive cancer and liver transplant surgery department and head of the surgery department.

e-mail: Farid_chehab@yahoo.fr

Institution: Department of General Surgery III, Ibn Rochd University

Hospital, Casablanca, Morocco. 